\title{
A Novel Approach to Solve Fully Fuzzy Linear Programming Problems with Modified Triangular Fuzzy Numbers
}

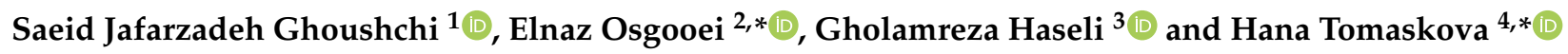 \\ 1 Faculty of Industrial Engineering, Urmia University of Technology, Urmia 57166, Iran; s.jafarzadeh@uut.ac.ir \\ 2 Faculty of Science, Urmia University of Technology, Urmia 57166, Iran \\ 3 Department of Management, Faculty of Economic, Management and Social Science, Shiraz University, \\ Shiraz 71345, Iran; ghr.haseli@gmail.com \\ 4 Faculty of Informatics and Management, University of Hradec Kralove, \\ 50006 Hradec Kralove, Czech Republic \\ * Correspondence: e.osgooei@uut.ac.ir (E.O.); hana.tomaskova@uhk.cz (H.T.)
}

Citation: Ghoushchi, S.J.; Osgooei,

E.; Haseli, G.; Tomaskova, H. A Novel Approach to Solve Fully Fuzzy Linear Programming Problems with Modified Triangular Fuzzy Numbers. Mathematics 2021, 9, 2937. https:// doi.org/10.3390/math9222937

Academic Editor: Aleksandr Rakhmangulov

Received: 12 October 2021

Accepted: 15 November 2021

Published: 18 November 2021

Publisher's Note: MDPI stays neutral with regard to jurisdictional claims in published maps and institutional affiliations.

Copyright: () 2021 by the authors. Licensee MDPI, Basel, Switzerland. This article is an open access article distributed under the terms and conditions of the Creative Commons Attribution (CC BY) license (https:// creativecommons.org/licenses/by/ $4.0 /)$.

\begin{abstract}
Recently, new methods have been recommended to solve fully fuzzy linear programming (FFLP) issues. Likewise, the present study examines a new approach to solve FFLP issues through fuzzy decision parameters and variables using triangular fuzzy numbers. The strategy, which is based on alpha-cut theory and modified triangular fuzzy numbers, is suggested to obtain the optimal fully fuzzy solution for real-world problems. In this method, the problem is considered as a fully fuzzy problem and then is solved by applying the new definition presented for the triangular fuzzy number to optimize decision variables and the objective function. Several numerical examples are solved to illustrate the above method.
\end{abstract}

Keywords: modified triangular fuzzy numbers; fuzzy decision variables; fully fuzzy linear programming; alpha-cut theory

\section{Introduction}

In the modern and competitive world, making the right, scientific-based, and timely decisions plays a very important and decisive role in the success or failure of organizations [1,2]. Uncertainty conditions and complexities of the decision-making processes have made decision makers use fuzzy theory, which is a suitable tool for management under uncertain conditions and making optimal decisions [3,4]. This theory was first proposed by Lotfi A. Zadeh [5] in 1965. Linear programming is an operational research tool that has been widely used for many years. Determining the parameters of fuzzy programming requires the opinions of decision makers; however, it cannot be determined accurately and definitively in most cases, because there is always ambiguity and uncertainty in the opinion of experts and decision makers. Fuzzy linear programming can also be explained as a fully fuzzy issue by considering all the decision parameters and variables of the problem in the fuzzy phase [6,7]. Therefore, various solution methods have been recommended to obtain the optimal solution of fuzzy linear programming. In the first method, Lotfi et al. [8] solved the problems by approximating the parameters to be as close as possible to symmetric triangular fuzzy numbers and the approximate optimal fuzzy solution. For this purpose, they solved a multiobjective linear programming model. Kumar et al. [9] used a linear ranking function to convert a fuzzy objective function to a certain state and, finally, obtained a certain optimal solution to solve fully fuzzy problems. Elsewhere, Ezzati et al. [10] solved problems using a new algorithm to convert the problem to multiobjective linear programming along with the lexicography method. This study examines a new approach to solve FFLP problems through fuzzy decision parameters and variables using triangular fuzzy numbers. The technique, which is based on alpha-cut theory and modified triangular fuzzy numbers, is suggested to obtain the optimal fully fuzzy solution for real-world 
problems. In this method, the problem is considered as a fully fuzzy problem and then is solved by applying the new definition presented for the triangular fuzzy number to optimize decision variables and the objective function. First, the parameters and variables of the model are written based on the modified triangular fuzzy numbers, and then the model is solved by considering the middle object as the objective function and the upper and lower objects as constraints. Based on this method, more accurate optimal results are obtained, and also the uncertainty of the model is reached.

The rest of this study proceeds as follows: Definitions of fuzzy numbers are described in Section 2. Section 3 presents a new technique to solve the FFLP issue. In Section 4, two simple examples and a case study about transportation optimization are provided to describe the new technique. In Section 5, the methods of Kumar et al. [9] and Ezzati et al. [10] are compared with the new method. Finally, the conclusion is given in Section 7.

\section{Preliminaries}

In this section, some of the foundations of the fuzzy theory are reviewed. The fuzzy theory defines the concept of membership function to express linguistic variables [5]. A membership function can be defined as $\mu_{A}(x): X \rightarrow[0,1]$ for set A. Membership amount $\mu_{A}(x)$ indicates the degree of dependence $x \in X$ in set $A$. The degree of conformity of member $x \in R$ to the intended concept of the set $\widetilde{A}$ or the degree of membership of any element $x \in R$ to a fuzzy set $\widetilde{A}$ is defined as the degree of acceptance of $x$ as a member of the fuzzy set $\widetilde{A}$.

Definition 1. A triangular fuzzy number $\widetilde{A}$ (as shown in Figure 1) is defined as a ternary number $(l, m, n)$ and its membership function is as follows:

$$
\mu_{A}(x)= \begin{cases}0 & x \leq l \\ \frac{x-l}{m-l} & l \leq x \leq m, \\ \frac{u-x}{u-m} & m \leq x \leq u \\ 0 & x \geq u\end{cases}
$$

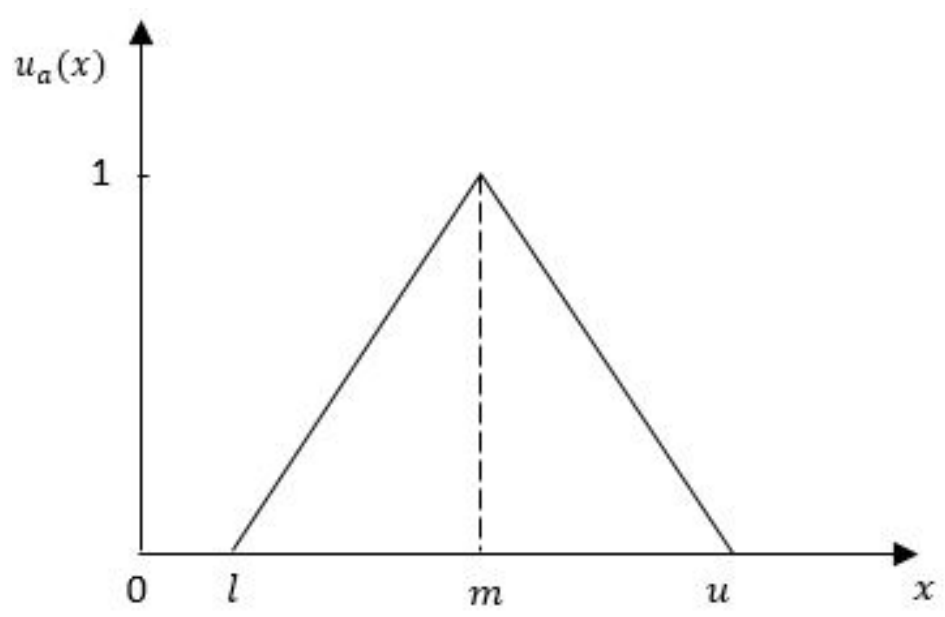

Figure 1. Triangular Fuzzy Number.

Definition 2 (Alpha-cut). The alpha cut for the fuzzy set $\widetilde{A}\left(A_{\alpha}\right)$ is a certain set that contains a set of elements, whose degree of membership is equal to or greater than the alpha value.

$$
A_{\alpha}=\left\{x \in X \mid \mu_{\widetilde{A}}(x) \geq \alpha\right\}
$$


where $\widetilde{A}=(l, m, n)$ is implied as a triangular fuzzy number, with its alpha cut being as follows:

$$
A_{\alpha}=[l+\alpha(m-l), u-\alpha(u-m)]
$$

In the following, new definitions to compare triangular fuzzy numbers based on Modified Triangular Fuzzy Numbers (TFNs) are introduced.

Definition 3 (Modified Triangular Fuzzy Numbers (MTFNs)). Assume $\widetilde{A}=(l, m, n)$ can be described as a triangular fuzzy number, the modified triangular fuzzy number (as shown in Figure 2) is defined as follows:

$$
\mu_{A_{\alpha}}(x)= \begin{cases}\frac{x-l^{\prime}}{m-l^{\prime}} & l^{\prime} \leq x \leq m \\ 1 & x=m \\ \frac{u^{\prime}-x}{u^{\prime}-m} & m \leq x \leq u^{\prime}\end{cases}
$$

where $l, m$, and $u$, respectively, represent the lower, modal, and upper value of the support of $\widetilde{A}$, all of which are crisp numbers $(-\infty<l \leq m \leq u<+\infty)$. Furthermore, $l^{\prime}$ and $u^{\prime}$, respectively, represent the lower and upper values of the modified triangular fuzzy numbers.

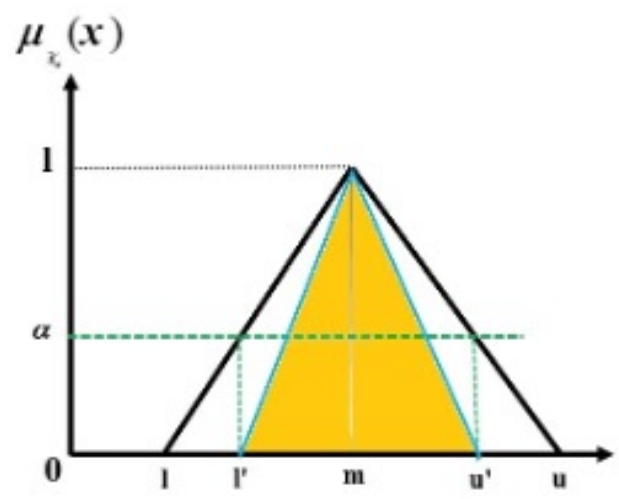

Figure 2. Modified triangular Fuzzy Number.

$$
\begin{gathered}
A_{\alpha}=\left(l^{\prime}, m, n^{\prime}\right) \\
l^{\prime}=l+\alpha(m-l), u^{\prime}=\alpha(u-m) \\
0 \leq \alpha \leq 1
\end{gathered}
$$

The fuzzy number $A_{\alpha}$ is obtained by the alpha cut in fuzzy number $A$, which leads to reducing the uncertainty in fuzzy number $A$.

Definition 4. Assume that $A_{\alpha}=\left(l_{1}+\alpha\left(m_{1}-l_{1}\right), m_{1}, u_{1}-\alpha\left(u_{1}-m_{1}\right)\right)$ and $B_{\alpha}=\left(l_{2}+\right.$ $\left.\alpha\left(m_{2}-l_{2}\right), m_{2}, u_{2}-\alpha\left(u_{2}-m_{2}\right)\right)$ are Modified Triangular Fuzzy Numbers.

Arithmetic operations are performed as follows:

$$
\begin{gathered}
A_{\alpha} \oplus B_{\alpha}=\left(l_{1}+\alpha\left(m_{1}-l_{1}\right)+l_{2}+\alpha\left(m_{2}-l_{2}\right)\right), m_{1}+m_{2} \\
\left.u_{1}-\alpha\left(u_{1}-m_{1}\right)+u_{2}-\alpha\left(u_{2}-m_{2}\right)\right) \\
A_{\alpha} \otimes B_{\alpha}=\left(\left(l_{1}+\alpha\left(m_{1}-l_{1}\right)\right)\left(l_{2}+\alpha\left(m_{2}-l_{2}\right)\right),\right. \\
m_{1} m_{2}, \\
\left.\left.u_{1}-\alpha\left(u_{1}-m_{1}\right)\right)\left(u_{2}-\alpha\left(u_{2}-m_{2}\right)\right)\right)
\end{gathered}
$$




$$
\begin{gathered}
\begin{array}{c}
A_{\alpha}-B_{\alpha}=\left(l_{1}+\alpha\left(m_{1}-l_{1}\right)-u_{2}+\alpha\left(u_{2}-m_{2}\right)\right), \\
m_{1}-m_{2}, \\
\left.u_{1}-\alpha\left(u_{1}-m_{1}\right)\right)-\left(l_{2}-\alpha\left(m_{2}-l_{2}\right)\right)
\end{array} \\
\begin{array}{r}
A_{\alpha} / B_{\alpha}=\left(l_{1}+\alpha\left(m_{1}-l_{1}\right) / u_{2}+\alpha\left(u_{2}-m_{2}\right)\right), m_{1} / m_{2}, \\
\left(u_{1}-\alpha\left(u_{1}-m_{1}\right)\right) /\left(l_{2}-\alpha\left(m_{2}-l_{2}\right)\right)
\end{array} \\
\begin{array}{r}
\left.\lambda A_{\alpha}=\lambda\left(l_{1}+\alpha\left(m_{1}-l_{1}\right)\right), m_{1}, u_{1}-\alpha\left(u_{1}-m_{1}\right)\right)= \\
=\left(\lambda\left(l_{1}+\alpha\left(m_{1}-l_{1}\right)\right), \lambda m_{1}, \lambda u_{1}-\alpha\left(u_{1}-m_{1}\right)\right), \lambda>0 \\
\left.\lambda A_{\alpha}=\lambda\left(l_{1}+\alpha\left(m_{1}-l_{1}\right)\right), m_{1}, u_{1}-\alpha\left(u_{1}-m_{1}\right)\right)= \\
\left.=\left(\lambda\left(u_{1}+\alpha\left(u_{1}-m_{1}\right)\right), \lambda m_{1}, \lambda l_{1}+\alpha\left(m_{1}-l_{1}\right)\right)\right), \lambda<0
\end{array}
\end{gathered}
$$

Definition 5. The two modified fuzzy numbers are compared as follows:

(i) $A_{\alpha} \approx B_{\alpha}$ if $l_{1}+\alpha\left(m_{1}-l_{1}\right)=l_{2}+\alpha\left(m_{2}-l_{2}\right), m_{1}=m_{2}, u_{1}-\alpha\left(u_{1}-m_{1}\right)=u_{2}-\alpha\left(u_{2}-\right.$ $\left.m_{2}\right)$

(ii) $A_{\alpha} \leq B_{\alpha}$ if $l_{1}+\alpha\left(m_{1}-l_{1}\right) \leq l_{2}+\alpha\left(m_{2}-l_{2}\right), m_{1} \leq m_{2}, u_{1}-\alpha\left(u_{1}-m_{1}\right) \leq u_{2}-\alpha\left(u_{2}-\right.$ $\left.m_{2}\right)$

(iii) $A_{\alpha} \geq B_{\alpha}$ if $l_{1}+\alpha\left(m_{1}-l_{1}\right) \geq l_{2}+\alpha\left(m_{2}-l_{2}\right), m_{1} \geq m_{2}, u_{1}-\alpha\left(u_{1}-m_{1}\right) \geq u_{2}-\alpha\left(u_{2}-\right.$ $\left.m_{2}\right)$

Definition 6. The modified triangular fuzzy number is nonnegative only if:

$$
A_{\alpha} \geq 0 \text { if } l_{1}+\alpha\left(m_{1}-l_{1}\right) \geq 0, \quad 0 \leq \alpha \leq 1
$$

Definition 7. Best non-fuzzy performance (BNP) can be calculated as follows:

$$
\begin{aligned}
& \operatorname{BNP}\left(A_{\alpha}\right)=\frac{((u-\alpha(u-m))-(l+\alpha(m-l))+(m-(l+\alpha(m-l)))}{3}+ \\
&+(l+\alpha(m-l))
\end{aligned}
$$

\section{Fully Fuzzy Linear Programming Issue (FFLP)}

Linear programming is one of the common and practical methods for operational research used to optimize problems. All parameters and variables are certain according to the linear programming assumptions. However, most of the problems of real-world cases are inaccurate and implicit. Therefore, this assumption of certainty of parameters and variables cannot solve problems accurately and obtain an optimal solution. Therefore, uncertainty is taken into consideration in the form of fuzzy numbers. In this way, the real-world problems are solved optimally via a new approach of FFLP in which all variables and parameters are uncertain [11]. The model is performed based upon Equation (1).

$$
\begin{array}{r}
\max (\min )\left(\widetilde{C}^{T} \otimes x\right) \\
\text { subject to: } \widetilde{A} \otimes x=\widetilde{b}
\end{array}
$$

$\widetilde{X}$ can be defined as a nonnegative triangular number.

Here, $\tilde{x}^{*}=\left(\left(x^{*}\right)^{l},\left(x^{*}\right)^{m},\left(x^{*}\right)^{u}\right)$ is an optimal solution if and only if the following conditions hold:

(i) $\tilde{x}$ is a nonnegative triangular fuzzy number;

(ii) $\widetilde{A} \otimes \widetilde{x}^{*}=\widetilde{b}$

(iii) $\forall \widetilde{x} \quad \widetilde{c} \widetilde{x} \leq \widetilde{c} \widetilde{x}^{*}$ (in minimization problems $\widetilde{c} \widetilde{x} \geq \widetilde{c}^{*}$ ) 
The flow chart of the proposed approach is illustrated in Figure 3.

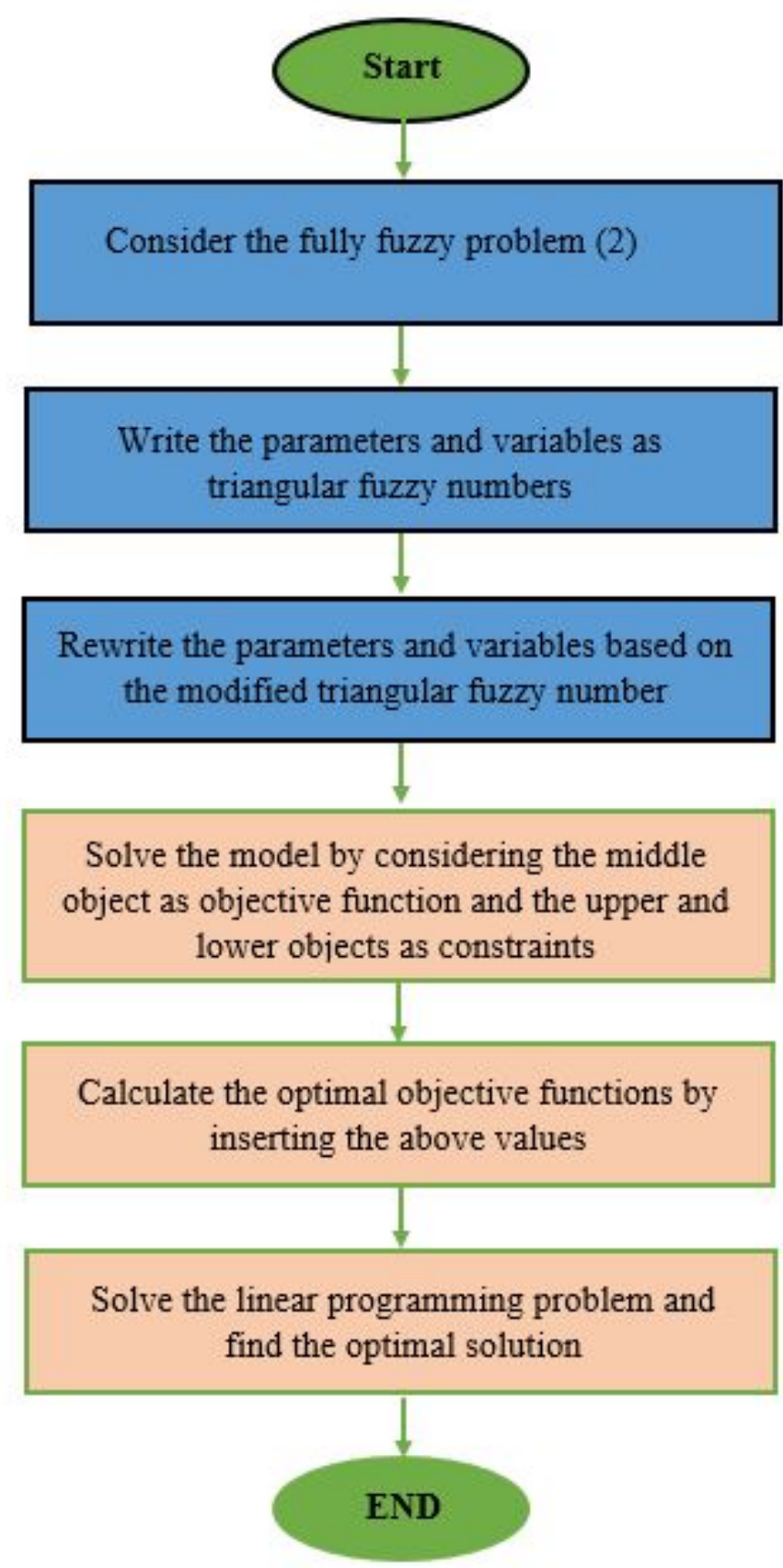

Figure 3. The flow chart of the proposed approach.

Step 1: According to the definitions, the problem is written as Equation (2):

$$
\begin{aligned}
& \max (\min ) \sum_{j=1}^{n} \widetilde{c}_{j} \otimes \widetilde{x}_{j} \\
& \text { subject to: } \\
& \sum_{j=1}^{n}\left(\widetilde{a}_{i j} \otimes \widetilde{x}_{j}\right)=\widetilde{b}_{i} \quad \forall i=1,2, \cdots, m
\end{aligned}
$$

$\widetilde{x}_{j}$ can be defined as a nonnegative triangular fuzzy number. 
Where $\widetilde{b}=\left[\widetilde{b}_{i}\right]_{m \times 1}, \quad \widetilde{A}=\left[\widetilde{a}_{i j}\right]_{m \times n}, \quad \widetilde{x}=\left[\widetilde{x}_{j}\right]_{n \times 1}, \quad \widetilde{C}^{T}=\left[\widetilde{c}_{j}\right]_{1 \times n}$, and $\widetilde{c}_{j}, \widetilde{x}_{j}, \widetilde{b}_{i} \in F(R)$.

Step 2: $\widetilde{c}_{j}, \widetilde{x}_{j}, \widetilde{a}_{i j}, \widetilde{b}_{i}$ are triangular fuzzy numbers as follows:

$$
\widetilde{c}_{j}=\left(c_{j}^{l}, c_{j}^{m}, c_{j}^{u}\right), \widetilde{x}_{j}=\left(x_{j}^{l}, x_{j}^{m}, x_{j}^{u}\right), \widetilde{a}_{i j}=\left(a_{i j}^{l}, a_{i j}^{m}, a_{i j}^{u}\right), \widetilde{b}_{i}=\left(b_{i}^{l}, b_{i}^{m}, b_{i}^{u}\right) .
$$

The FFLP issue of stage 1 is implied as Equation (3):

$$
\max (\min ) \sum_{j=1}^{n}\left(\left(c_{j}^{l}, c_{j}^{m}, c_{j}^{u}\right) \otimes\left(x_{j}^{l}, x_{j}^{m}, x_{j}^{u}\right)\right)
$$

subject to:

$$
\sum_{j=1}^{n}\left(\left(a_{i j}^{l}, a_{i j}^{m}, a_{i j}^{u}\right) \otimes\left(x_{j}^{l}, x_{j}^{m}, x_{j}^{u}\right)\right)=\left(b_{i}^{l}, b_{i}^{m}, b_{i}^{u}\right) \quad \forall i=1,2, \cdots, m
$$

$\left(x_{j}^{l}, x_{j}^{m}, x_{j}^{u}\right)$ can be defined as a nonnegative triangle fuzzy number.

Step 3: According to Definition 3, all decision parameters and variables are written based on the modified triangular fuzzy number as Equation (4).

$$
\begin{aligned}
& \widetilde{c}_{j}=\left(\left(c_{j}^{l}+\alpha\left(c_{j}^{m}-c_{j}^{l}\right), c_{j}^{m}, c_{j}^{u}-\alpha\left(c_{j}^{u}-c_{j}^{m}\right)\right), \widetilde{x}_{j}=\left(x_{j}^{l}+\alpha\left(x_{j}^{m}-x_{j}^{l}\right), x_{j}^{m}, x_{j}^{u}-\alpha\left(x_{j}^{u}-x_{j}^{m}\right)\right)\right. \\
& \widetilde{a}_{i j}=\left(a_{i j}^{l}+\alpha\left(a_{i j}^{m}-a_{i j}^{l}\right), a_{i j}^{m}, a_{i j}^{u}-\alpha\left(a_{i j}^{u}-a_{i j}^{m}\right)\right), \widetilde{b}_{i}=\left(b_{i}^{l}+\alpha\left(b_{i}^{m}-b_{i}^{l}\right), b_{i}^{m}, b_{i}^{u}-\alpha\left(b_{i}^{u}-b_{i}^{m}\right)\right) \\
& \max (\min ) \sum_{j=1}^{n}\left(c_{j}^{l}+\alpha\left(c_{j}^{m}-c_{j}^{l}\right), c_{j}^{m}, c_{j}^{u}-\alpha\left(c_{j}^{u}-c_{j}^{m}\right)\right)\left(x_{j}^{l}+\alpha\left(x_{j}^{m}-x_{j}^{l}\right), x_{j}^{m}, x_{j}^{u}-\alpha\left(x_{j}^{u}-x_{j}^{m}\right)\right)
\end{aligned}
$$

subject to:

$$
\begin{aligned}
& \sum_{j=1}^{n}\left(\left(a_{i j}^{l}+\alpha\left(a_{i j}^{m}-a_{i j}^{l}\right)\right)\left(x_{j}^{l}+\alpha\left(x_{j}^{m}-x_{j}^{l}\right)\right)\right)=b_{i}^{l}+\alpha\left(b_{i}^{m}-b_{i}^{l}\right) \forall i=1,2, \cdots, m \\
& \sum_{j=1}^{n}\left(a_{i j}^{m} x_{j}^{m}\right)=b_{j}^{m} \quad \forall i=1,2, \cdots, m \\
& \sum_{j=1}^{n}\left(\left(a_{i j}^{u}-\alpha\left(a_{i j}^{u}-a_{i j}^{m}\right)\right)\left(x_{j}^{l}+\alpha\left(x_{j}^{m}-x_{j}^{l}\right)\right)\right)=b_{i}^{u}-\alpha\left(b_{i}^{u}-b_{i}^{m}\right) \quad \forall i=1,2, \cdots, m \\
& x_{j}^{m}-\left(x_{j}^{l}+\alpha\left(x_{j}^{m}-x_{j}^{l}\right)\right) \geq 0, \quad\left(x_{j}^{u}-\alpha\left(x_{j}^{u}-x_{j}^{m}\right)\right)-x_{j}^{m} \geq 0, \quad x_{j}^{l}+\alpha\left(x_{j}^{m}-x_{j}^{l}\right) \geq 0 .
\end{aligned}
$$

Step 4: To solve the above model, the value of the objective function is considered equal to $\sum_{j=1}^{n} c_{j}^{m} x_{j}^{m}$, and then two constraints of $\left(c_{j}^{l}+\alpha\left(c_{j}^{m}-c_{j}^{l}\right)\right)\left(x_{j}^{l}+\alpha\left(x_{j}^{m}-x_{j}^{l}\right)\right) \leq\left(c_{j}^{m} x_{j}^{m}\right)$ and $\left(c_{j}^{m} x_{j}^{m}\right) \leq\left(c_{j}^{u}+\alpha\left(c_{j}^{u}-c_{j}^{m}\right)\right)\left(x_{j}^{u}+\alpha\left(x_{j}^{u}-x_{j}^{m}\right)\right)$ are applied to the model. According to Definition 4, the model in stage 3 is implied as Equation (5): 
$\max (\min ) \sum_{j=1}^{n} c_{j}^{m} x_{j}^{m}$

subject to:

$\left(c_{j}^{l}+\alpha\left(c_{j}^{m}-c_{j}^{l}\right)\right)\left(x_{j}^{l}+\alpha\left(x_{j}^{m}-x_{j}^{l}\right)\right) \leq\left(c_{j}^{m} x_{j}^{m}\right)$

$\left(c_{j}^{m} x_{j}^{m}\right) \leq\left(c_{j}^{u}+\alpha\left(c_{j}^{u}-c_{j}^{m}\right)\right)\left(x_{j}^{u}+\alpha\left(x_{j}^{u}-x_{j}^{m}\right)\right)$

$\sum_{j=1}^{n}\left(\left(a_{i j}^{l}-\alpha\left(a_{i j}^{m}-a_{i j}^{l}\right)\right)\left(x_{j}^{l}+\alpha\left(x_{j}^{m}-x_{j}^{l}\right)\right)\right)=b_{i}^{l}-\alpha\left(b_{i}^{m}-b_{i}^{l}\right) \quad \forall i=1,2, \cdots, m$

$\sum_{j=1}^{n}\left(a_{i j}^{m} x_{j}^{m}\right)=b_{j}^{m} \quad \forall i=1,2, \cdots, m$

$\sum_{j=1}^{n}\left(\left(a_{i j}^{u}-\alpha\left(a_{i j}^{u}-a_{i j}^{m}\right)\right)\left(x_{j}^{u}+\alpha\left(x_{j}^{u}-x_{j}^{m}\right)\right)\right)=b_{i}^{u}-\alpha\left(b_{i}^{u}-b_{i}^{m}\right) \quad \forall i=1,2, \cdots, m$

$x_{j}^{m}-\left(x_{j}^{l}+\alpha\left(x_{j}^{m}-x_{j}^{l}\right)\right) \geq 0, \quad\left(x_{j}^{u}-\alpha\left(x_{j}^{u}-x_{j}^{m}\right)\right)-x_{j}^{m} \geq 0, x_{j}^{l}+\alpha\left(x_{j}^{m}-x_{j}^{l}\right) \geq 0$.

Step 5: The above problem is solved, and the optimal values of $\left(x_{j}^{l}+\alpha\left(x_{j}^{m}-x_{j}^{l}\right)\right), x_{j}^{m}$ and $\left(x_{j}^{u}-\alpha\left(x_{j}^{u}-x_{j}^{m}\right)\right)$ are obtained.

Step 6: By inserting the above values, the value $\widetilde{x}_{j}=\left(x_{j}^{l}, x_{j}^{m}, x_{j}^{u}\right)$ is obtained.

Step 7: The value of the optimal objective function is calculated by inserting $\widetilde{x}_{j}$ into the $\operatorname{model} \sum_{j=1}^{n}\left(\widetilde{c}_{j} \otimes \widetilde{x}_{j}\right)$.

\section{Numerical Examples}

For example, if $A=(1,3,5), \alpha=0.3$, then the modified triangular fuzzy number is calculated as follows:

$$
A_{\alpha}=(1+0.3(3-1), 3,5-0.3(5-3))=(1.6,3,4.4)
$$

In this section, the proposed model is described using numerical examples:

Example 1. Kumar et al. [9]: The following FFLP problem is solved using the proposed model as follows:

$$
\max \left((1,2,3) \otimes x_{1} \oplus(1,2,3) \otimes x_{2}\right)
$$

Subject to:

$(0,1,2) \otimes x_{1} \oplus(1,2,3) \otimes x_{2}=(2,10,24)$,

$(1,2,3) \otimes x_{1} \oplus(0,1,2) \otimes x_{2}=(1,8,21)$,

$x_{1}, x_{2}$ is a nonnegative triangle fuzzy number.

Where $x_{1}=\left(x_{1}^{l}, x_{1}^{m}, x_{1}^{u}\right), x_{2}=\left(x_{2}^{l}, x_{2}^{m}, x_{2}^{u}\right)$

According to step 4, problem 6 is rewritten as (7):

$$
\max \left(2 x_{1}^{m}+3 x_{2}^{m}\right)
$$


Subject to:

$$
\begin{aligned}
& \left((1+\alpha(2-1))\left(x_{1}^{l}+\alpha\left(x_{1}^{m}-x_{1}^{l}\right)\right)+(2+\alpha(3-2))\left(x_{2}^{l}+\alpha\left(x_{2}^{m}-x_{2}^{l}\right)\right) \leq\left(2 x_{1}^{m}+3 x_{2}^{m}\right)\right. \\
& \left(2 x_{1}^{m}+3 x_{2}^{m}\right) \leq(3-\alpha(3-2))\left(x_{1}^{u}-\alpha\left(x_{1}^{u}-x_{1}^{m}\right)\right)+(4-\alpha(4-3))\left(x_{2}^{u}-\alpha\left(x_{2}^{u}-x_{2}^{m}\right)\right) \\
& (0+\alpha(1-0))\left(x_{1}^{l}+\alpha\left(x_{1}^{m}-x_{1}^{l}\right)\right)+(1+\alpha(2-1))\left(x_{2}^{l}+\alpha\left(x_{2}^{m}-x_{2}^{l}\right)\right)=2+\alpha(10-2) \\
& 1 x_{1}^{m} 2 x_{2}^{m}=10 \\
& (2-\alpha(2-1))\left(x_{1}^{u}-\alpha\left(x_{1}^{u}-x_{1}^{l}\right)\right)+(3-\alpha(3-2))\left(x_{2}^{u}-\alpha\left(x_{2}^{u}-x_{2}^{m}\right)\right)=24-\alpha(24-10) \\
& (1+\alpha(2-1))\left(x_{1}^{l}+\alpha\left(x_{1}^{m}-x_{1}^{l}\right)\right)+(0+\alpha(1-0))\left(x_{2}^{l}+\alpha\left(x_{2}^{m}-x_{2}^{l}\right)\right)=1+\alpha(8-1) \\
& 2 x_{1}^{m}+1 x_{2}^{m}=8 \\
& (3-\alpha(3-2))\left(x_{1}^{u}-\alpha\left(x_{1}^{u}-x_{1}^{m}\right)\right)+\left(2-\alpha(2-1)\left(x_{2}^{u}-\alpha\left(x_{2}^{u}-x_{2}^{m}\right)\right)=21-\alpha(21-8)\right. \\
& x_{1}^{m}-\left(x_{1}^{l}+\alpha\left(x_{1}^{m}-x_{1}^{l}\right)\right) \geq 0, \quad\left(x_{1}^{u}-\alpha\left(x_{1}^{u}-x_{1}^{m}\right)\right)-x_{1}^{m} \geq 0, \\
& x_{2}^{m}-\left(x_{2}^{l}+\alpha\left(x_{2}^{m}-x_{2}^{l}\right)\right) \geq 0, \quad\left(x_{2}^{u}-\alpha\left(x_{2}^{u}-x_{2}^{m}\right)\right)-x_{2}^{m} \geq 0
\end{aligned}
$$

Considering $\alpha=0.3$, the answer is obtained as follows: The optimal solution is:

$$
\begin{aligned}
& x_{1}^{l}=1.56, x_{1}^{m}=2, x_{1}^{u}=3.2, \\
& x_{2}^{l}=2.56, x_{2}^{m}=4, x_{2}^{u}=6.2 . \\
& x_{1}=(1.56,2,3.2), x_{2}=(2.56,4,6.2) \\
& \left(c x^{*}\right)_{\text {Proposed method }}=\left(\left(c x^{*}\right)^{l},\left(c x^{*}\right)^{m},\left(c x^{*}\right)^{u}\right)=(6.68,16,34.4)
\end{aligned}
$$

Using Kumar's method, the results of the model are obtained as follows:

$$
\begin{aligned}
& x_{1}=(1,2,3), \quad x_{2}=(2,4,6) \\
& \left(c x^{*}\right)_{\text {Kumar's method }}=\left(\left(c x^{*}\right)^{l},\left(c x^{*}\right)^{m},\left(c x^{*}\right)^{u}\right)=(5,16,33)
\end{aligned}
$$

Using Ezzati's method, the results of the model are obtained as follows:

$$
\begin{aligned}
& x_{1}=(1,2,3), x_{2}=(2,4,6) \\
& \left(c x^{*}\right)_{\text {Ezzati's method }}=\left(\left(c x^{*}\right)^{l},\left(c x^{*}\right)^{m},\left(c x^{*}\right)^{u}\right)=(5,16,33)
\end{aligned}
$$

Example 2. Ezzati et al. [10]: Consider the following FFLP issue as (8):

$\max \left(\widetilde{c}^{T} \otimes x\right)$

Subject to: $\widetilde{A} \widetilde{x}=\widetilde{b}$

Where the values of $\widetilde{A}, \widetilde{x}$, and $\widetilde{c}$ are as follows:

$$
\begin{aligned}
& \widetilde{c}=\left[\begin{array}{c}
(10,15,17) \\
(10,16,20) \\
(10,14,17) \\
(10,12,14)
\end{array}\right], \widetilde{A}=\left[\begin{array}{cccc}
(8,10,13) & (10,11,13) & (9,12,13) & (11,15,17) \\
(12,14,16) & (14,18,19) & (14,17,20) & (13,14,18)
\end{array}\right] \\
& \widetilde{b}=\left[\begin{array}{c}
(271.75,411.75,573.75) \\
(385.5,539.5,759.5)
\end{array}\right]
\end{aligned}
$$


$\max \left((10,15,17) \otimes x_{1} \oplus(10,16,20) \otimes x_{2} \oplus(10,14,17) \otimes x_{3} \oplus(10,12,14) \otimes x_{4}\right)$

Subject to:

$(8,10,13) \otimes x_{1} \oplus(10,11,13) \otimes x_{2} \oplus(9,12,13) \otimes x_{3} \oplus(11,15,17) \otimes x_{4}=$ $=(271.75,411.75,573.75)$

$(12,14,16) \otimes x_{1} \oplus(14,18,19) \otimes x_{2} \oplus(14,17,20) \otimes x_{3} \oplus(13,14,18) \otimes x_{4}=$ $=(385.5,539.5,759.5)$

$x_{1}, x_{2}, x_{3}, x_{4}$ are nonnegative triangular fuzzy numbers

Where $x_{1}=\left(x_{1}^{l}, x_{1}^{m}, x_{1}^{u}\right), x_{2}=\left(x_{2}^{l}, x_{2}^{m}, x_{2}^{u}\right), x_{3}=\left(x_{3}^{l}, x_{3}^{m}, x_{3}^{u}\right), x_{4}=\left(x_{4}^{l}, x_{4}^{m}, x_{4}^{u}\right)$ According to step 4, problem (9) is rewritten as follows:

$$
\begin{aligned}
& \max \left(15 x_{1}^{m}+16 x_{2}^{m}+14 x_{3}^{m}+12 x_{4}^{m}\right) \\
& \text { Subject to: } \\
& (10+\alpha(15-10)) \times\left(x_{1}^{l}+\alpha\left(x_{1}^{m}-x_{1}^{l}\right)\right)+(10+\alpha(16-10)) \times \\
& \times\left(x_{2}^{l}+\alpha\left(x_{2}^{m}-x_{2}^{l}\right)\right)+(10+\alpha(14-10)) \times\left(x_{3}^{l}+\alpha\left(x_{3}^{m}-x_{3}^{l}\right)\right)+ \\
& +(10+\alpha(12-10)) \times\left(x_{4}^{l}+\alpha\left(x_{4}^{m}-x_{4}^{l}\right)\right) \leq \\
& \leq 15 x_{1}^{m}+16 x_{2}^{m}+14 x_{3}^{m}+12 x_{4}^{m} \leq \\
& \leq(17+\alpha(17-15)) \times\left(x_{1}^{u}+\alpha\left(x_{1}^{u}-x_{1}^{m}\right)\right)+(20+\alpha(20-16)) \times \\
& \times\left(x_{2}^{u}+\alpha\left(x_{2}^{u}-x_{2}^{m}\right)\right)+(17+\alpha(17-14)) \times\left(x_{3}^{u}+\alpha\left(x_{3}^{u}-x_{3}^{m}\right)\right)+ \\
& +(14+\alpha(14-12)) \times\left(x_{4}^{u}+\alpha\left(x_{4}^{u}-x_{4}^{m}\right)\right) \\
& (8+\alpha(10-8)) \times\left(x_{1}^{l}+\alpha\left(x_{1}^{m}-x_{1}^{l}\right)\right)+(10+\alpha(11-10)) \times\left(x_{2}^{l}+\alpha\left(x_{2}^{m}-x_{2}^{l}\right)\right)+ \\
& +(9+\alpha(12-9)) \times\left(x_{3}^{l}+\alpha\left(x_{3}^{m}-x_{3}^{l}\right)\right)+(11+\alpha(15-11)) \times\left(x_{4}^{l}+\alpha\left(x_{4}^{m}-x_{4}^{l}\right)\right)= \\
& =271.75+\alpha(411.75-271.75) \\
& 10 x_{1}^{m}+11 x_{2}^{m}+12 x_{3}^{m}+15 x_{4}^{m}=411.75 \\
& (13-\alpha(13-10)) \times\left(x_{1}^{u}+\alpha\left(x_{1}^{u}-x_{1}^{m}\right)\right)+(13-\alpha(13-11)) \times\left(x_{2}^{u}+\alpha\left(x_{2}^{u}-x_{2}^{m}\right)\right)+ \\
& +(13-\alpha(13-12)) \times\left(x_{3}^{u}+\alpha\left(x_{3}^{u}-x_{3}^{m}\right)\right)+(17-\alpha(17-15)) \times\left(x_{4}^{u}+\alpha\left(x_{4}^{u}-x_{4}^{m}\right)\right)= \\
& =573.75-\alpha(573.75-411.75) \\
& (12+\alpha(14-12)) \times\left(x_{1}^{l}+\alpha\left(x_{1}^{m}-x_{1}^{l}\right)\right)+(14+\alpha(18-14)) \times\left(x_{2}^{l}+\alpha\left(x_{2}^{m}-x_{2}^{l}\right)\right)+ \\
& +(14+\alpha(17-14)) \times\left(x_{3}^{l}+\alpha\left(x_{3}^{m}-x_{3}^{l}\right)\right)+(13+\alpha(14-13)) \times\left(x_{4}^{l}+\alpha\left(x_{4}^{m}-x_{4}^{l}\right)\right)= \\
& =385.5+\alpha(539.5-385.5) \\
& 14 x_{1}^{m}+18 x_{2}^{m}+17 x_{3}^{m}+14 x_{4}^{m}=539.5 \\
& (16-\alpha(16-14)) \times\left(x_{1}^{u}+\alpha\left(x_{1}^{u}-x_{1}^{m}\right)\right)+(19-\alpha(19-18)) \times\left(x_{2}^{u}+\alpha\left(x_{2}^{u}-x_{2}^{m}\right)\right)+ \\
& +(17-\alpha(20-17)) \times\left(x_{3}^{u}+\alpha\left(x_{3}^{u}-x_{3}^{m}\right)\right)+(14-\alpha(18-14)) \times\left(x_{4}^{u}+\alpha\left(x_{4}^{u}-x_{4}^{m}\right)\right)= \\
& =759.5+\alpha(759.5-539.5) \\
& x_{1}^{m}-\left(x_{1}^{l}+\alpha\left(x_{1}^{m}-x_{1}^{l}\right)\right) \geq 0, \quad\left(x_{1}^{u}-\alpha\left(x_{1}^{u}-x_{1}^{m}\right)\right)-x_{1}^{m} \geq 0, \\
& x_{2}^{m}-\left(x_{2}^{l}+\alpha\left(x_{2}^{m}-x_{2}^{l}\right)\right) \geq 0, \quad\left(x_{2}^{u}-\alpha\left(x_{2}^{u}-x_{2}^{m}\right)\right)-x_{2}^{m} \geq 0, \\
& x_{3}^{m}-\left(x_{3}^{l}+\alpha\left(x_{3}^{m}-x_{3}^{l}\right)\right) \geq 0, \quad\left(x_{3}^{u}-\alpha\left(x_{3}^{u}-x_{3}^{m}\right)\right)-x_{3}^{m} \geq 0, \\
& x_{4}^{m}-\left(x_{4}^{l}+\alpha\left(x_{4}^{m}-x_{4}^{l}\right)\right) \geq 0, \quad\left(x_{4}^{u}-\alpha\left(x_{4}^{u}-x_{4}^{m}\right)\right)-x_{4}^{m} \geq 0 \\
& \alpha=0.7
\end{aligned}
$$

By solving the above model, the results of the problem are obtained as follows:

$$
\begin{aligned}
& x_{1}^{l}=17.59, x_{1}^{m}=17.59, x_{1}^{u}=17.59, x_{2}^{l}=0, x_{2}^{m}=2.7, x_{2}^{u}=9.75, \\
& x_{3}^{l}=7.20, x_{3}^{m}=9, x_{3}^{u}=9, x_{4}^{l}=6.54, x_{4}^{m}=6.54, x_{4}^{u}=6.54
\end{aligned}
$$


The optimal answer to Equation (10) is as follows:

$$
\begin{aligned}
& x_{1}=\left(x_{1}^{l}, x_{1}^{m}, x_{1}^{u}\right)=(17.59,17.59,17.59), x_{2}=\left(x_{2}^{l}, x_{2}^{m}, x_{2}^{u}\right)=(0,2.7,9.75), \\
& x_{3}=\left(x_{3}^{l}, x_{3}^{m}, x_{3}^{u}\right)=(7.20,9,9), x_{4}=\left(x_{4}^{l}, x_{4}^{m}, x_{4}^{u}\right)=(6.54,6.54,6.54) \\
& \left(c x^{*}\right)_{\text {Proposed method }}=\left(\left(c x^{*}\right)^{l},\left(c x^{*}\right)^{m},\left(c x^{*}\right)^{u}\right)=(313.3,511.53,738.59)
\end{aligned}
$$

Using Kumar's method, the results of the model are obtained as follows:

$$
\begin{aligned}
& x_{1}=\left(x_{1}^{l}, x_{1}^{m}, x_{1}^{u}\right)=(15.28,15.28,15.28), x_{2}=\left(x_{2}^{l}, x_{2}^{m}, x_{2}^{u}\right)=(2.4,2.4,9.1), \\
& x_{3}=\left(x_{3}^{l}, x_{3}^{m}, x_{3}^{u}\right)=(6,11.25,11.25), x_{4}=\left(x_{4}^{l}, x_{4}^{m}, x_{4}^{u}\right)=(6.49,6.49,6.49) \\
& \left(c x^{*}\right)_{\text {Kumar's method }}=\left(\left(c x^{*}\right)^{l},\left(c x^{*}\right)^{m},\left(c x^{*}\right)^{u}\right)=(301.83,503.23,724.15)
\end{aligned}
$$

Using Ezzati's method, the results of the model are obtained as follows:

$$
\begin{aligned}
& x_{1}=\left(x_{1}^{l}, x_{1}^{m}, x_{1}^{u}\right)=(17.27,17.27,17.27), x_{2}=\left(x_{2}^{l}, x_{2}^{m}, x_{2}^{u}\right)=(2.16,2.16,2.16), \\
& x_{3}=\left(x_{3}^{l}, x_{3}^{m}, x_{3}^{u}\right)=(4.64,9.97,16.36), x_{4}=\left(x_{4}^{l}, x_{4}^{m}, x_{4}^{u}\right)=(6.36,6.36,6.36) \\
& \left(c x^{*}\right)_{\text {Ezzati's method }}=\left(\left(c x^{*}\right)^{l},\left(c x^{*}\right)^{m},\left(c x^{*}\right)^{u}\right)=(304.58,509.79,704.37)
\end{aligned}
$$

Example 3. Dali Company plans to develop its market to other countries. It has four distribution centers in Taipei, Kaohsiung, Chiayi, and Taichung and three plants in Hsinchu, Touliu, and Changhua. Table 1 shows the unit transportation costs between these centers in USD.

Table 1. Summary information of potential suppliers, demand and supply in 000 dozen bottles, transportation costs between centers in USD.

\begin{tabular}{cccccc}
\hline \multirow{2}{*}{ Source } & \multicolumn{4}{c}{ Destination } & \multirow{2}{*}{ Supply } \\
& Taipei & Kaohsiung & Chiayi & B & (7.2,8,8.8) \\
\hline Changhua & $(18.8,20,22)$ & $(8,10,10.6)$ & $(20.4,22,24)$ & $(8,10,10.8)$ & $(7.24)$ \\
Touliu & $(6,8,8.8)$ & $(10,12,13)$ & $(18.2,20,22)$ & $(14,15,16)$ & $(12,14,16)$ \\
Hsinchu & $(14,15,16)$ & $(7.8,10,10.8)$ & $(9.6,12,13)$ & $(18.4,20,21)$ & $(10.2,12,13.8)$ \\
Demand & $(7.8,9,10.2)$ & $(6.5,8,9.5)$ & $(8.9,10,11.1)$ & $(6.2,7,7.8)$ & \\
\hline
\end{tabular}

Given that the parameters of the problem are inaccurate and uncertain, regarding the issue of shipping costs, the problem is solved using the mentioned approach.

$$
\begin{aligned}
& \min \left((8,10,10.8) \otimes x_{11} \oplus(20.4,22,24) \otimes x_{12} \oplus(8,10,10.6) \otimes x_{13} \oplus(18.8,20,22) \otimes x_{14} \oplus\right. \\
& \oplus(14,15,16) \otimes x_{21} \oplus(18.2,20,22) \otimes x_{22} \oplus(10,12,13) \otimes x_{23} \oplus(6,8,8.8) \otimes x_{24} \oplus \\
& \left.\oplus(18.4,20,21) \otimes x_{31} \oplus(9.6,12,13) \otimes x_{32} \oplus(7.8,10,10.8) \otimes x_{33} \oplus(14,15,16) \oplus x_{34}\right)
\end{aligned}
$$

Subject to:

$x_{11} \oplus x_{12} \oplus x_{13} \oplus x_{14}=(7.2,8,8.8), \quad x_{31} \oplus x_{32} \oplus x_{33} \oplus x_{34}=(10.2,12,13.8)$,

$x_{21} \oplus x_{22} \oplus x_{23} \oplus x_{24}=(12,14,16), \quad x_{11} \oplus x_{21} \oplus x_{31}=(6.2,7,7.8)$,

$x_{12} \oplus x_{22} \oplus x_{32}=(8.9,10,11.1), \quad x_{13} \oplus x_{23} \oplus x_{33}=(6.5,8,9.5)$,

$x_{14} \oplus x_{24} \oplus x_{34}=(7.8,9,10.2)$

where:

$$
\begin{aligned}
& x_{11}=\left(x_{11}^{l}, x_{11}^{m}, x_{11}^{u}\right), x_{12}=\left(x_{12}^{l}, x_{12}^{m}, x_{12}^{u}\right), x_{13}=\left(x_{13}^{l}, x_{13}^{m}, x_{13}^{u}\right), x_{14}=\left(x_{14}^{l}, x_{14}^{m}, x_{14}^{u}\right), \\
& x_{21}=\left(x_{21}^{l}, x_{21}^{m}, x_{21}^{u}\right), x_{22}=\left(x_{22}^{l}, x_{22}^{m}, x_{22}^{u}\right), x_{23}=\left(x_{23}^{l}, x_{23}^{m}, x_{23}^{u}\right), x_{24}=\left(x_{24}^{l}, x_{24}^{m}, x_{24}^{u}\right), \\
& x_{31}=\left(x_{31}^{l}, x_{31}^{m}, x_{31}^{u}\right), x_{32}=\left(x_{32}^{l}, x_{32}^{m}, x_{32}^{u}\right), x_{33}=\left(x_{33}^{l}, x_{33}^{m}, x_{33}^{u}\right), x_{34}=\left(x_{34}^{l}, x_{34}^{m}, x_{34}^{u}\right)
\end{aligned}
$$


The results of the model are obtained as follows:

$$
\begin{aligned}
& x_{11}=\left(x_{11}^{l}, x_{11}^{m}, x_{11}^{u}\right)=(6.2,7,7), x_{12}=\left(x_{12}^{l}, x_{12}^{m}, x_{12}^{u}\right)=(0,0,0), \\
& x_{13}=\left(x_{13}^{l}, x_{13}^{m}, x_{13}^{u}\right)=(1,1,1), x_{14}=\left(x_{14}^{l}, x_{14}^{m}, x_{14}^{u}\right)=(0,0,0), \\
& x_{21}=\left(x_{21}^{l}, x_{21}^{m}, x_{21}^{u}\right)=(0,0,0), x_{22}=\left(x_{22}^{l}, x_{22}^{m}, x_{22}^{u}\right)=(0,0,0), \\
& x_{23}=\left(x_{23}^{l}, x_{23}^{m}, x_{23}^{u}\right)=(4.2,5,5.8), x_{24}=\left(x_{24}^{l}, x_{24}^{m}, x_{24}^{u}\right)=(7.8,9,10.2), \\
& x_{31}=\left(x_{31}^{l}, x_{31}^{m}, x_{31}^{u}\right)=(0,0,0), x_{32}=\left(x_{32}^{l}, x_{32}^{m}, x_{32}^{u}\right)=(8.9,10,11.1), \\
& x_{33}=\left(x_{33}^{l}, x_{33}^{m}, x_{33}^{u}\right)=(1.3,2,2.7), x_{34}=\left(x_{34}^{l}, x_{34}^{m}, x_{34}^{u}\right)=(0,0,0) \\
& \left(c x^{*}\right)_{\text {Proposed method }}=\left(\left(c x^{*}\right)^{l},\left(c x^{*}\right)^{m},\left(c x^{*}\right)^{u}\right)=(241.98,352,453.5)
\end{aligned}
$$

Using Kumar's method, the results of the model are obtained as follows:

$$
\begin{aligned}
& x_{11}=\left(x_{11}^{l}, x_{11}^{m}, x_{11}^{u}\right)=(6.2,7,7.8), x_{12}=\left(x_{12}^{l}, x_{12}^{m}, x_{12}^{u}\right)=(0,0,0), \\
& x_{13}=\left(x_{13}^{l}, x_{13}^{m}, x_{13}^{u}\right)=(1,1,1), x_{14}=\left(x_{14}^{l}, x_{14}^{m}, x_{14}^{u}\right)=(0,0,0), \\
& x_{21}=\left(x_{21}^{l}, x_{21}^{m}, x_{21}^{u}\right)=(0,0,0), x_{22}=\left(x_{22}^{l}, x_{22}^{m}, x_{22}^{u}\right)=(0,0,0), \\
& x_{23}=\left(x_{23}^{l}, x_{23}^{m}, x_{23}^{u}\right)=(4.2,5,5.8), x_{24}=\left(x_{24}^{l}, x_{24}^{m}, x_{24}^{u}\right)=(7.8,9,10.2), \\
& x_{31}=\left(x_{31}^{l}, x_{31}^{m}, x_{31}^{u}\right)=(0,0,0), x_{32}=\left(x_{32}^{l}, x_{32}^{m}, x_{32}^{u}\right)=(8.9,10,11.1), \\
& x_{33}=\left(x_{33}^{l}, x_{33}^{m}, x_{33}^{u}\right)=(1.3,2,2.7), x_{34}=\left(x_{34}^{l}, x_{34}^{m}, x_{34}^{u}\right)=(0,0,0) \\
& \left(c x^{*}\right)_{\text {Kumar's method }}=\left(\left(c x^{*}\right)^{l},\left(c x^{*}\right)^{m},\left(c x^{*}\right)^{u}\right)=(241.98,352,433.46)
\end{aligned}
$$

Employing Ezzati's technique, the results of the model are obtained as follows:

$$
\begin{aligned}
& x_{11}=\left(x_{11}^{l}, x_{11}^{m}, x_{11}^{u}\right)=(6.2,7,7.8), x_{12}=\left(x_{12}^{l}, x_{12}^{m}, x_{12}^{u}\right)=(0,0,0), \\
& x_{13}=\left(x_{13}^{l}, x_{13}^{m}, x_{13}^{u}\right)=(1,1,1), x_{14}=\left(x_{14}^{l}, x_{14}^{m}, x_{14}^{u}\right)=(0,0,0), \\
& x_{21}=\left(x_{21}^{l}, x_{21}^{m}, x_{21}^{u}\right)=(0,0,0), x_{22}=\left(x_{22}^{l}, x_{22}^{m}, x_{22}^{u}\right)=(0,0,1.1), \\
& x_{23}=\left(x_{23}^{l}, x_{23}^{m}, x_{23}^{u}\right)=\left(4.2,5,5.9, x_{24}=\left(x_{24}^{l}, x_{24}^{m}, x_{24}^{u}\right)=(7.8,9,9),\right. \\
& x_{31}=\left(x_{31}^{l}, x_{31}^{m}, x_{31}^{u}\right)=(0,0,0.8), x_{32}=\left(x_{32}^{l}, x_{32}^{m}, x_{32}^{u}\right)=(8.9,10,10), \\
& x_{33}=\left(x_{33}^{l}, x_{33}^{m}, x_{33}^{u}\right)=(1.3,2,2.6), x_{34}=\left(x_{34}^{l}, x_{34}^{m}, x_{34}^{u}\right)=(0,0,0) \\
& \left(c x^{*}\right)_{\text {Ezzati's method }}=\left(\left(c x^{*}\right)^{l},\left(c x^{*}\right)^{m},\left(c x^{*}\right)^{u}\right)=(241.98,352,465.18)
\end{aligned}
$$

According to Definition 5 and comparing the proposed method results with the results of Kumar and Ezzati, it can be concluded that the results of this method are more accurate than Kumar's Method and close to Ezzati's. The results of this method are compared with the current methods, as shown in Table 2. 
Table 2. Comparison of proposed method with other existing methods.

\begin{tabular}{lccc}
\hline & Kumar & Ezatti & $\begin{array}{c}\text { Proposed Approach } \\
\boldsymbol{\alpha}=\mathbf{0 . 5}\end{array}$ \\
\hline$x_{11}=\left(x_{11}^{l}, x_{11}^{m}, x_{11}^{u}\right)$ & $(6.2,7,7.8)$ & $(6.2,7,7)$ & $(6.2,7,7)$ \\
$x_{12}=\left(x_{12}^{l}, x_{12}^{m}, x_{12}^{u}\right)$ & $(0,0,0)$ & $(0,0,0)$ & $(0,0,0)$ \\
$x_{13}=\left(x_{13}^{l}, x_{13}^{m}, x_{13}^{u}\right)$ & $(1,1,1)$ & $(1,1,1)$ & $(1,1,1)$ \\
$x_{14}=\left(x_{14}^{l}, x_{14}^{m}, x_{14}^{u}\right)$ & $(0,0,0)$ & $(0,0,0.8)$ & $(0,0,0.8)$ \\
$x_{21}=\left(x_{21}^{l}, x_{21}^{m}, x_{21}^{u}\right)$ & $(0,0,0)$ & $(0,0,0)$ & $(0,0,0)$ \\
$x_{22}=\left(x_{22}^{l}, x_{22}^{m}, x_{22}^{u}\right)$ & $(0,0,0)$ & $(0,0,0.1)$ & $(0,0,0.1)$ \\
$x_{23}=\left(x_{23}^{l}, x_{23}^{m}, x_{23}^{u}\right)$ & $(4.2,5,5.8)$ & $(4.2,5,5.9)$ & $(4.2,5,5.9)$ \\
$x_{24}=\left(x_{24}^{l}, x_{24}^{m}, x_{24}^{u}\right)$ & $(7.8,9,10.2)$ & $(7.8,9,9)$ & $(7.8,9,9)$ \\
$x_{31}=\left(x_{31}^{l}, x_{31}^{m}, x_{31}^{u}\right)$ & $(0,0,0)$ & $(0,0,0.8)$ & $(0,0,0.8)$ \\
$x_{32}=\left(x_{32}^{l}, x_{32}^{m}, x_{32}^{u}\right)$ & $(8.9,10,11.1)$ & $(8.9,10,10)$ & $(8.9,10,10)$ \\
$x_{33}=\left(x_{33}^{l}, x_{33}^{m}, x_{33}^{u}\right)$ & $(1.3,2,2.7)$ & $(1.3,2,2.6)$ & $(1.3,2,2.6)$ \\
$x_{34}=\left(x_{34}^{l}, x_{34}^{m}, x_{34}^{u}\right)$ & $(0,0,0)$ & $(0,0,0.4)$ & $(0,0,0.4)$ \\
$\left(c x^{*}\right)=\left(\left(c x^{*}\right)^{l},\left(c x^{*}\right)^{m},\left(c x^{*}\right)^{u}\right)$ & $(241.98,352,433.46)$ & $(241.98,352,465.18)$ & $(241.98,352,453.5)$ \\
\hline
\end{tabular}

\section{Sensitivity Analysis}

The results of this method are given in Table 3 for Example 3 with various alpha values. Since different answers were obtained, a decision maker can properly choose the desired answer, i.e., it increases the decision-making power.

Table 3. Results of example 3 with different alpha values.

\begin{tabular}{cc}
\hline$\alpha$ & $c x^{*}=\left(\left(c x^{*}\right)^{l},\left(c x^{*}\right)^{m},\left(c x^{*}\right)^{u}\right)$ \\
\hline 0 & $(241.98,352,461.42)$ \\
0.1 & $(241.98,352,454.46)$ \\
0.2 & $(241.98,352,442.28)$ \\
0.3 & $(241.98,352,433.46)$ \\
0.4 & $(241.98,352,454.46)$ \\
0.5 & $(241.98,352,453.50)$ \\
0.6 & $(241.98,352,442.28)$ \\
0.7 & $(241.98,352,440.04)$ \\
0.8 & $(241.98,352,433.46)$ \\
0.9 & $(241.98,352,452.22)$ \\
\hline
\end{tabular}

\section{The Advantages of the Proposed Model over Other Methods}

Kumar et al. [9] presented an optimal solution for fully fuzzy problems employing the linear ranking function and arithmetic operations. In this method, the fuzzy nature of the objective function is ignored and calculated as a certain value, while the objective function is considered fuzzy in the technique presented in this paper. Moreover, Ezzati et al. [10] presented a certain optimal solution by converting the objective function into a multiobjective function. In this method, the answer is obtained through fewer steps and the computing speed is high.

According to the obtained results, it can be concluded that the proposed technique leads to more accurate optimal results. Furthermore, the uncertainty of the model is reduced due to the new definition presented for the triangular fuzzy number and different alpha values. Therefore, there are different optimal answers available for the decision maker.

\section{Conclusions}

This study presents a new approach to solve FFLP issues and obtains the optimal solution under equal constraints. After rewriting the parameters and variables based on the modified triangular fuzzy numbers, the middle object is considered as the objective function and the upper and lower objects as constraints. Then, the FFLP model as a 
linear model is solved. Based on the proposed method and using the modified triangular fuzzy numbers, a more accurate optimal result is obtained than with Kumar's method. In Kumar's method, using the ranking function to invert the fuzzy concept into certain valued, some data are missing, and so the solution is not accurate enough. This is the benefit and advantage of the proposed method in comparison with Kumar's. To validate the efficiency of the technique, the obtained results were compared with those obtained using Kumar's and Ezzati's methods.

Author Contributions: S.J.G.: conceptualization, writing—original draft, methodology, validation; E.O.: writing — original draft, formal analysis, methodology, investigation; G.H.: formal analysis, writing - review and editing, visualization; H.T.: investigation, visualization; validation. All authors have read and agreed to the published version of the manuscript.

Funding: The APC was funded by FIM UHK Excellence Project 2021: Decision Support Systems: Principles and Applications 3.

Institutional Review Board Statement: Not applicable.

Informed Consent Statement: Not applicable.

Conflicts of Interest: The authors declare no conflict of interest.

\section{References}

1. Ghoushchi, S.J.; Dorosti, S.; Khazaeili, M.; Mardani, A. Extended approach by using best-worst method on the basis of importance-necessity concept and its application. Appl. Intell. 2021, 51, 8030-8044. [CrossRef]

2. Lin, Z.; Ayed, H.; Bouallegue, B.; Tomaskova, H.; Jafarzadeh Ghoushchi, S.; Haseli, G. An Integrated Mathematical Attitude Utilizing Fully Fuzzy BWM and Fuzzy WASPAS for Risk Evaluation in a SOFC. Mathematics 2021, 9, 2328. [CrossRef]

3. Ghoushchi, S.J.; Khazaeili, M. G-Numbers: Importance-necessity concept in uncertain environment. Int. J. Manag. Fuzzy Syst. 2019, 5, 27-32. [CrossRef]

4. Sun, C.; Li, S.; Deng, Y. Determining weights in multi-criteria decision making based on negation of probability distribution under uncertain environment. Mathematics 2020, 8, 191. [CrossRef]

5. Zadeh, L.A.; Klir, G.J.; Yuan, B. Fuzzy Sets, Fuzzy Logic, and Fuzzy Systems: Selected Papers; World Scientific: Singapore, 1996; Volume 6.

6. Das, S.K.; Mandal, T.; Edalatpanah, S. A mathematical model for solving fully fuzzy linear programming problem with trapezoidal fuzzy numbers. Appl. Intell. 2017, 46, 509-519. [CrossRef]

7. Sharma, U.; Aggarwal, S. Solving fully fuzzy multi-objective linear programming problem using nearest interval approximation of fuzzy number and interval programming. Int. J. Fuzzy Syst. 2018, 20, 488-499. [CrossRef]

8. Lotfi, F.H.; Allahviranloo, T.; Jondabeh, M.A.; Alizadeh, L. Solving a full fuzzy linear programming using lexicography method and fuzzy approximate solution. Appl. Math. Model. 2009, 33, 3151-3156. [CrossRef]

9. Kumar, A.; Kaur, J.; Singh, P. A new method for solving fully fuzzy linear programming problems. Appl. Math. Model. 2011, 35, 817-823. [CrossRef]

10. Ezzati, R.; Khorram, E.; Enayati, R. A new algorithm to solve fully fuzzy linear programming problems using the MOLP problem. Appl. Math. Model. 2015, 39, 3183-3193. [CrossRef]

11. Allahviranloo, T.; Salahshour, S.; Khezerloo, M. Maximal-and minimal symmetric solutions of fully fuzzy linear systems. J. Comput. Appl. Math. 2011, 235, 4652-4662. [CrossRef] 